MS11.04.03 SOLID-STATE REACTIONS OF INORGANIC COMPOUNDS. Elena V. Boldyreva". Institute of Solid State Chemistry Siberian Department of the Russian Academy of Sciences. Kutateladze, 18, Novosibirsk, 128, 630128 Russia

There are so many different types of "solid-state reactions of inorganic compounds", that it may seem impossible to cover such a topic within a 20 minute lecture. What can formation of a complex oxide in the powder mixture of simple metal oxides have in common with the photo-isomerization in coordination compounds, with an intercallation - reaction, or with the decomposition of metal azides, crystal hydrates, or $\mathrm{KMnO}_{4}$ ? The present contribution is aimed to give an answer to this question. Looking for something common between absolutely different types of solid-state reactions of inorganic compounds, we shall concentrate our attention on the role of crystal structure in a solid-state transformation.

We shall start with the static aspects of the effect of crystal structure on the solid-state transformations. The crystal structure will be considered as a "frame" determining the mutual juxtaposition of the reacting chemical species, as well as the size, the shape, and the mutual orientation of the channels serving for the transport of ions; atoms, or molecules. Examples of epitaxial and topotactic solid-state reactions will be given, the methods of "soft chemistry" and the "precursor technique" will be discussed.

We shall proceed then with considering the dynamic aspects of the effect of the crystal structure on the solid-state transformations. We shall demonstrate that the strain induced in the crystal structure by the reaction can be the main factor, determining the kinetics and the spatial pattern of the reaction, as well as the structure, the morphology, and thus, the properties of solid products.

"In 1995-1996 - a Humboldt Fellow at the Institute of Physical Chemistry, Marburg University.

\section{MS11.04.04 THE EFFECTS OF COHERENCY ON PHASE}

EQUILIBRIA. J. B. Cohen, Department of Materials Science and Engineering, Robert R., McCormick School of Engineering and Applied Science, Northwestern, University, Evanston, IL 60208

Recent theories have predicted large effects on both phase composition and volume fraction if a precipitating phase is coherent, but there has been no satisfactory experimental verification. Furthermore, these effects vary with overall composition of an alloy. For example, in the presence of coherency stresses, the compositions of the phases in a two-phase region are not constant, as they are for incoherent equilibria. Such coherent phase equilibria were examined experimentally in a Ni-24 at.\% V alloy, using an in-situ high-temperature $\mathrm{X}$-ray attachment mounted on a diffractometer. Peak positions of the a phase shifted to higher $2 q$ during the transition from coherency to incoherency, indicating that the vanadium content in the coherent a phase is higher than that of the incoherent phase. There was no significant peak shift observed for the 400 peak of the $b$ phase. These peak shifts are expected from these recent theories on the role of coherency stresses on phase equilibria. (In order to calculate the coherent phase diagrams from these theories for comparison with experiment, the elastic constants and misfit versus temperature were also measured.) Other experiments will also be described.

This research was funded by the National Science Foundation (Grant DMR 8417961) and was performed with D. K.Na and P. W. Voorhees.
MS11.04.05 SOLIO STATE POLYMERIZATION REACTIONS INALKALI SALTS OF HALOGENOCARBOXYLIC ACDDS Oliver Herzberg, Holger Kirschnick and Matthias Epple, Institute of Inorganic and Applied Chemistry, University of Hamburg, Martin-Luther- King-Platz 6, D-20146 Hamburg, Germany

Thermal oligomerization and polymerization takes place in alkali salts of halogenocarboxylic acids, e.g.: $\mathrm{NaOOC}-\mathrm{CH}(\mathrm{Cl})$ $\mathrm{CH}_{2}-\mathrm{CH}_{3} \rightarrow \mathrm{NaCl}+1 / \mathrm{n}\left(-\mathrm{OOCCH}\left(\mathrm{CH}_{2} \mathrm{CH}_{3}\right)\right.$-)n This reaction leads to pharmaceutically and technically interesting oligomers and polymers which were characterized by gel permeation chromatography (GPC). These polyesters of hydroxycarboxylic acids are easily biodegradable in the body and the environment. The precursor salts are metastable and sometimes difficult to prepare, because some are highly unstable towards elimination of $\mathrm{HCl}$ to alkenoic acid salts. Some were prepared as for the first time, although reported as "unstable at room temperature" in the literature. The salts were characterized by powder diffractometry, thermal analysis and solid-state NMR. The above polymerization reaction was followed by in situ X-ray powder diffractometry and thermal analysis in order to gain insight into the mechanism of this solid-state reaction. It is remarkably exothermic which underscores the metastable character of the educt substances.

M.Epple, L. Troeger, J. Chem. Soc., Dalton Trans., 1996, 11-16.

MS11.04.06 THE KINETICS OF THE PRESSUREINDUCED B1-B2 PHASE TRANSFORMATION IN RUBIDIUM IODIDE. A. R. Rezai-Fard and J. Anwar, King's College London, Manresa Road, London SW3 6LX, UK; S. M. Clark, Daresbury Laboratory, Daresbury, Warrington, WA4 4AD, UK

The kinetics of the pressure-induced $\mathrm{B} 1(\mathrm{Fm} 3 \mathrm{~m})-\mathrm{B} 2(\mathrm{Pm} 3 \mathrm{~m})$ phase transition of rubidium iodide have been investigated in a large-volume high-pressure cell using energy dispersive $\mathrm{X}$-ray powder diffraction. The kinetics were followed at various excess pressures under isobaric conditions. The transformation did not always go to completion, with the fraction transformed as a function of time tending to plateau. Both the rate and extent of transformation were generally higher at higher excess pressures. This behaviour, which has been observed before (Hamaya and Akimoto 1981; Dassler 1990), can be explained by considering that the powder sample is characterised by nucleation sites with a distribution of activation energies. Thus crystallites containing sites with lower activation energy transform at low excess pressures whilst those with higher activation energies remain unreacted. The kinetics were analysed considering only the fraction of material that had transformed. Various kinetic models were fitted and the Avrami equation with $n=1$ was found to be the best, though in absolute terms just acceptable.

Finally, the fraction transformed with time in terms of the new $(\alpha)$ phase and the parent $(\beta)$ phase did not always add up to one as expected. After consideration and exclusion of all other possibilities this problem was attributed to the existence of an amorphous phase. This amorphous phase is not considered to be an intermediate but thought to coexist. It interconverts with the crystalline phases during phase transition.

References:

Dassler R, Hight Temp.-High Press., 22, 599-612, (1990)

Hamaya $\mathrm{N}$ and Akimoto S, High Temp.-High Press., 13, 347-358, (1981) 